Gut, 1988, 29, 580-587

\title{
Lactosylceramide in inflammatory bowel disease: a biochemical study
}

\author{
C R STEVENS, V G OBERHOLZER, J A WALKER-SMITH, \\ AND A D PHILLIPS
}

From the Queen Elizabeth Hospital for Children, London

\begin{abstract}
SUMmARY A simple technique for isolating lipids from small pieces of tissue was applied to mucosal biopsies and samples of resected intestine from patients with inflammatory bowel disease. Scanning densitometry of two dimensional chromatograms showed increased concentrations of the membrane associated glycosphingolipid lactosylceramide in Crohn's disease, on comparison with ulcerative colitis $(\mathbf{p}<\mathbf{0 . 0 1})$, or controls $(\mathbf{p}<\mathbf{0 . 0 1})$. This indicates either that normal glycosphingolipid metabolism is altered, or that a novel source of lactosylceramide is present in the inflamed mucosa of patients with Crohn's disease.
\end{abstract}

A previous ultrastructural study from this hospital observed small round microvilli and desmosome associated bodies in small and large intestinal mucosal biopsies from patients with inflammatory bowel disease.' Preliminary histochemical analysis, suggesting that they may be plasmalogen in nature (Lewis et al, unpublished observations), prompted chromatographic analysis of the lipid content of mucosal extracts from such patients. Initial work showed no abnormality in plasmalogens, however, another type of lipid, lactosylceramide, was found to

Address for correspondence: A D Phillips, Electron Microscopy, Queen Elizabeth Hospital for Children. Hackney Road, London E2 8PS

Received for publication 2 November 1987. be greatly increased in Crohn's disease. This paper describes a preliminary visual assessment and a subsequent estimation by scanning densitometry of the presence of lactosylceramide in inflammatory bowel disease.

\section{Methods}

PATIENTS

A total of 65 patients (57 children, eight adults) were studied, 32 provided samples for an initial visual assessment of lipid content and 33 for an objective study using scanning densitometry. These were divided into four clinical categories as shown in the Table. Categorisation of the patients was achieved

Table Details of patients and samples

\begin{tabular}{|c|c|c|c|c|c|c|c|c|c|}
\hline \multirow[b]{2}{*}{ Diagnosis } & \multirow[b]{2}{*}{ Cases } & \multicolumn{4}{|c|}{$B X /$ Resec site } & \multirow{2}{*}{$\begin{array}{l}\text { Resec } \\
\text { samples }\end{array}$} & \multirow[b]{2}{*}{ Adult } & \multirow[b]{2}{*}{ Male } & \multirow[b]{2}{*}{ Female } \\
\hline & & $T I$ & $L C$ & $R C$ & $R M$ & & & & \\
\hline \multicolumn{10}{|l|}{ Visual assessment } \\
\hline Crohn's disease & 10 & 6 & 2 & 1 & 1 & 3 & 2 & 4 & 6 \\
\hline Ulcerative colitis & 11 & 0 & 2 & 3 & 6 & 2 & 4 & 5 & 6 \\
\hline Control & 11 & 3 & 3 & 4 & 1 & 0 & 1 & 3 & 8 \\
\hline Totals & 32 & 9 & 7 & 8 & 8 & 5 & 7 & 12 & 20 \\
\hline \multicolumn{10}{|c|}{ Estimation by scanning densitometry } \\
\hline Crohn's disease & 12 & 8 & 1 & 2 & 1 & 2 & 0 & 8 & 4 \\
\hline Ulcerative colitis & 10 & 0 & 5 & 4 & 1 & 1 & 1 & 4 & 6 \\
\hline Control & 8 & 4 & 2 & 2 & 0 & 0 & () & 2 & 6 \\
\hline Indeterminate colitis & 3 & 0 & 3 & 0 & 0 & 0 & 0) & 2 & 1 \\
\hline Totals & 33 & 12 & 11 & 8 & 2 & 3 & 1 & 16 & 17 \\
\hline
\end{tabular}


using diagnoses made from a combination of clinical, radiological, endoscopic, and histological criteria. ${ }^{2}$

All patients were examined endoscopically to investigate clinical symptoms compatible with inflammatory bowel disease, and a provisional diagnosis could sometimes be made from the site and appearance of inflammation.

A final diagnosis is usually made from a histological examination of endoscopic biopsies. Positive stigmata of ulcerative colitis are mucosal crypt distortion and crypt abscesses, mucus depletion, and a uniformity of inflammation. The lesion in Crohn's disease, however, characteristically consists of patchy, focal inflammation, which may be transmural, with non-caseating granulomata in $40-70 \%$ of cases. ${ }^{3+}$ The cases of indeterminate colitis have been impossible to categorise as either definite Crohn's disease or ulcerative colitis by the means outlined above. ${ }^{5}$

Twenty two patients had Crohn's disease of which four had ileal disease only, two colonic disease only, and 16 had ileal and colonic disease. Three patients were adults. The ages of the children ranged from 8 years 8 months to 17 years 11 months, with a median age of 14 years 3 months. The medical records of 18 of these patients were available. Six patients were not receiving medication at the time of biopsy whereas 12 were receiving Salazopyrin and/or Prednisolone. Duration of disease ranged from one month to nine years with most patients studied two to four years after onset of symptoms. Eleven of 16 erythrocyte sedimentation rate (ESR) measurements before colonoscopy were abnormal ( $>20 \mathrm{~mm} /$ hour).

Sixteen of the 21 patients with ulcerative colitis were children with a median age of 13 years 1 month (range 6 years 2 months to 15 years 6 months). The medical records of 14 of these patients were available. Two patients were on Salazopyrin. Twelve patients were receiving Prednisolone and of these, five were also on Salazopyrin and three on Mesalazine. Antidiarrhoeal drugs were in use in four cases (three Loperamide, one Lomotil). Duration of disease ranged from 7 months to 10 years, and the majority of patients were studied one to four years after onset of symptoms. Five of nine ESR measurements taken before colonoscopy were above normal limits.

The three children with indeterminate colitis had ages of 12 years 2 months, 10 years 6 months and 9 years 2 months; their disease durations were four years, two years six months, and 10 months respectively. All three were receiving Prednisolone at the time of biopsy.

The control group consisted of 19 children (median age 8 years 2 months, range 11 months to 14 years) and one adult. All underwent colonoscopy to investi- gate clinical symptoms compatible with the diagnosis of inflammatory bowel disease, but no endoscopic or histological abnormality could be detected. One patient was receiving cimetidine treatment for a duodenal ulcer; another patient was prescribed local steroids for mouth ulcers and antihistamine for a maculopapular rash.

\section{BIOPSY MATERIAI.}

Mucosal tissue was obtained from biopsy specimens or from surgical resections (Table). The biopsies were taken during routine colonoscopy or sigmoidoscopy, and included samples from all parts of the distal bowel to $20 \mathrm{~cm}$ into the terminal ileum. Samples were frozen immediately. Most of the tissue was processed within two to 24 hours. Mucosa from resected gut was sometimes available at the time of surgery but generally was used after being frozen at $-70^{\circ}$ for several years. Samples from patients with inflammatory bowel disease used in either study (Table) were taken from inflamed areas.

To supplement the main study, chromatographic analysis, followed by visual assessment, was carried out on lipid extracts from: (1) areas of the bowel from five of the patients with Crohn's disease which showed no endoscopic nor histological abnormality (terminal ileum $[n=1]$, right colon $[n=1]$, left colon $[n=3]) ;(2)$ small intestinal biopsies of the fourth part of the duodenum from three patients with active coeliac disease; and (3) left colonic biopsies of two patients with a microscopic colitis.

\section{ISOLATION OF HUMAN PERIPHERAL BLOOD}

\section{NEUTROPHILS}

Whole blood was taken from healthy, volunteer laboratory staff and treated with ethylenediaminetetraacetic acid (EDTA). Erythrocytes were sedimented after mixing 0.6 volumes of $6 \%$ dextran in $0.9 \%$ sodium chloride with 1 volume whole blood. The total number of leucocytes in the supernatant was obtained using a ZF Coulter counter, a differential cell count done, and the number of neutrophils/ millilitre of supernatant estimated. Neutrophils were then isolated as described by Ferrante $e t$ a $l^{\circ}$ for lipid extraction. A Lieshman's stained smear indicated a neutrophil layer of $98 \%$ purity.

EXTRACTION OF PHOSPHOLIPID/

GLYCOSPHINGOLIPID FRACTION FROM TISSUE AND NEUTROPHIL SAMPLES

All solvents used were Analar grade and were mixed on a volume basis. The extraction technique used is a modification of that described by Bligh and Dyer.? Samples of tissue (up to approximately $6 \mathrm{mg}$ in weight) and comparable volumes of blood neutrophils were homogenised at room temperature in 1.9 
$\mathrm{ml}$ methanol/chloroform/water mixture (10:5:4) for four minutes in a hand homogeniser (Baird and Tatlock $10 \mathrm{~mm}$ diameter). A further $0.5 \mathrm{ml}$ chloroform was added and homogenisation repeated for four minutes to enhance extraction. Distilled water, $0.5 \mathrm{ml}$, was added to separate the chloroform extract from the aqueous methanol phase. The resulting bilayer was clarified by centrifugation at $500 \mathrm{~g}$ for 10 minutes and the extract (lower phase) taken to dryness under a stream of nitrogen in a heated $\left(37^{\circ} \mathrm{C}\right)$ block (Grant Instruments).

TWO DIMENSIONAL THIN-LAYER CHROMATOGRAPHY (2D-TLC)

Chromatographic sheets $(10 \mathrm{~cm} \times 10 \mathrm{~cm}$ Silica Gel G 60 plastic backed. Merck AG, no $5748 \mathrm{BDH}$ ) were heat activated at $80^{\circ} \mathrm{C}$ for at least one hour before sample application. Dried lipid extracts were redissolved in $25 \mu \mathrm{l}$ chloroform and aliquots applied to one corner of each sheet. The total extract from small biopsy samples was applied whereas larger biopsies and pieces of resection supplied more than enough extract to give a clear chromatogram so that only fractions of the total extract were applied. Samples were first run in a solvent system of chloroform/methanol/toluene/water $\quad$ (70:35:25:5). On completion, and after drying in air, the sheets were turned through $90^{\circ}$ and stood in an alkaline solvent system chloroform $/ \mathrm{methanol} /$ toluene $/ 5 \mathrm{M}$ aqueous ammonia (70:35:25:5). Lipid spots were visualised by charring the sheets in a fan assisted oven at $110^{\circ} \mathrm{C} \pm 1^{\circ} \mathrm{C}$, after dipping in $5 \mathrm{M} \mathrm{H}_{2} \mathrm{SO}_{4} /$ methanol $(2: 8)$. The chromatograms were examined on a light box immediately after charring.

\section{LIPID IDENTIFICATION}

The lipid spots seen on 2D-TLC were first categorised by their reaction with spray reagents - for example, molybdenum blue, which detects phospholipids, and orcinol and diphenylamine, which detect glycolipids. Further elucidation was achieved by comparing spot migration rates determined by TLC with previously observed values for known lipids. ${ }^{x-11}$ Finally, commercially available standards of the main phospholipids and glycolipids were obtained and individually cochromatographed with the extracted lipid fraction. The identity of each lipid was given as that of its comigrating standard after careful comparison of spot colour and mobility. A standard of lactosylceramide was not commercially available so a sample isolated from a patient with Crohn's disease was sent for confirmation of identity to Dr B A Macher, University of California, ${ }^{7}$ who in turn, kindly provided a positive standard.

Lipid spots were isolated for identification by scraping the appropriate area of silica gel from a chromatogram then eluting the resultant powdered silica with chloroform/methanol (2:1) dropwise through a syringe plugged with fine glass wool. The elutant was taken to dryness as before and stored at $-20^{\circ} \mathrm{C}$.

QUALITATIVE ESTIMATION OF

LACTOSYLCERAMIDE

A visual assessment of the two closely travelling purple-grey lactosylceramide spots was made as absent - that is, not visible, faintly present, and strongly present.

ESTIMATION OF LACTOSYLCERAMIDE BY

\section{SCANNING DENSITOMETRY}

Thirty three tissue samples (Table) were coded before extraction and chromatography as described above. Chromatograms were processed under constant conditions of temperature and timing to standardise the inherent variables of spot intensity, background, and degree of charring and fading. Values for the spot densities of lactosylceramide and phosphatidylethanolamine were obtained using a Joyce Loebl Chromoscan 3 densitometer (Joyce-Loebl, Gateshead, Tyne and Wear). The Chromoscan 3 is a high performance densitometer under microprocessor control (Intel 8085). The raster scan has a resolution of $5 \mu \mathrm{m}$. All chromatograms were scanned not more than three hours after charring at $530 \mathrm{~nm}$ wavelength, with absorbance set at 1 , using a $1 \mathrm{~mm}^{2}$ aperture in raster mode.

The small amount of tissue available did not allow a measurement of lactosylceramide per weight of biopsy or weight of protein. Lactosylceramide (LC) was therefore expressed as a density ratio to that of a reference phospholipid present in the same extract. Phosphatidylethanolamine (PE) was chosen as the reference because it constitutes $30-35 \%$ of membrane phospholipid ${ }^{78}$ and exists in different tissues in comparable quantities. " Assuming these statements to be valid for the lipid content of diseased and healthy intestinal mucosa, we considered the amount of phosphatidylethanolamine present on a chromatogram to represent the amount of tissue which provided the spotted extract. Phosphatidylethanolamine appeared in all the analysed extracts as a compact, well separated spot which travelled to a position unaffected by interference bands arising from impurities in the silica gel itself. These factors facilitated the accurate and reproducible measurement of phosphatidylethanolamine density by scanning densitometry.

To assess the reproducibility of the LC/PE ratio graded dilutions (1:1-1:30) of an extract from a patient with Crohn's disease were chromatographed and their densitometric data analysed. Also, equal 


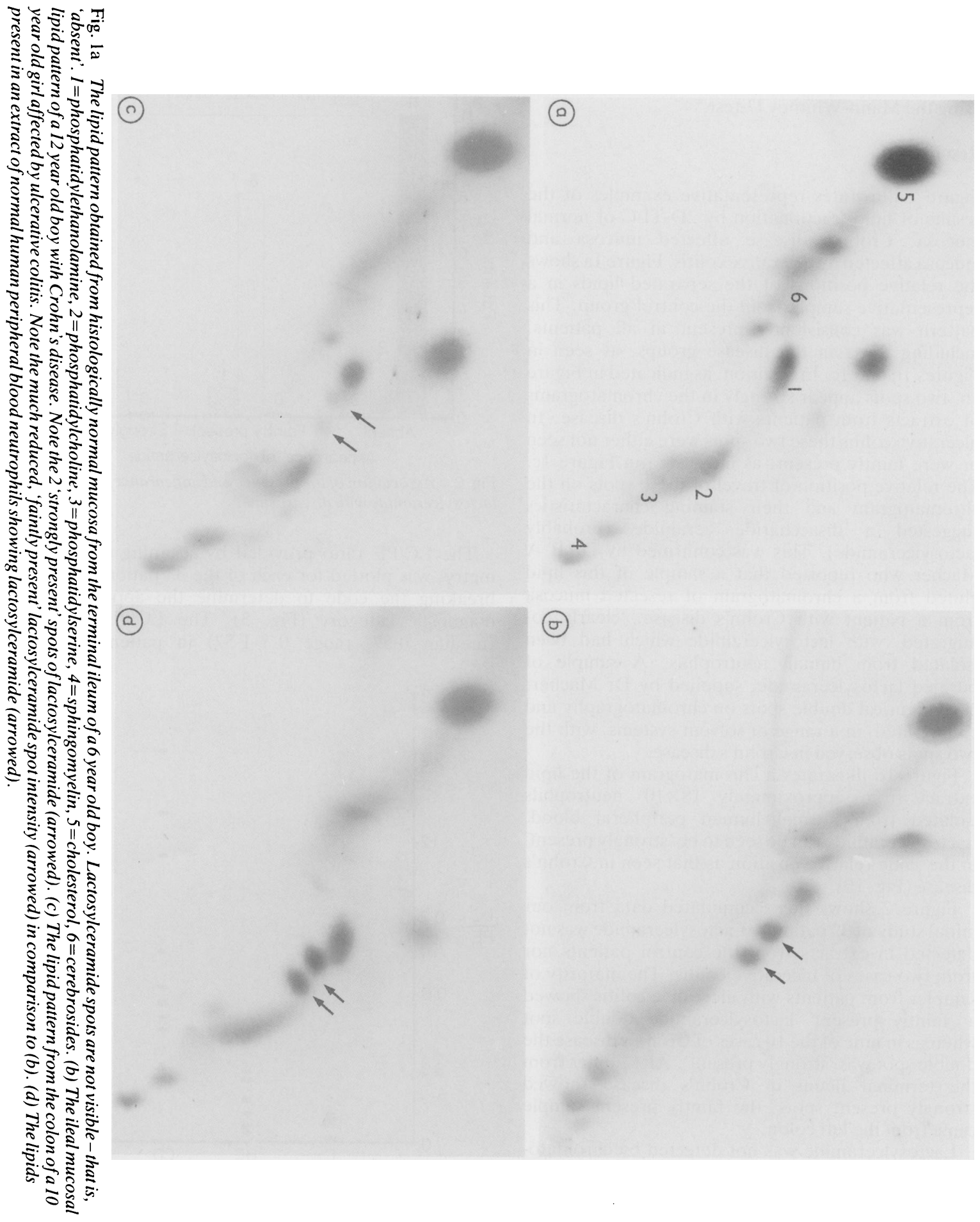


aliquots of homogeneous neutrophil samples from a normal adult donor were processed separately to assess the reproducibility of the extraction technique.

Statistical analysis of the results was performed using the Mann-Whitney U-test.

\section{Results}

Figure 1 illustrates representative examples of the results of lipid fractionation by 2D-TLC of normal mucosa, Crohn's disease affected mucosa and mucosa affected by ulcerative colitis. Figure la shows the relative positions of the separated lipids in a representative sample from the control group. This pattern was consistently present in all patients, including those in the disease groups, as seen in Figures $1 \mathrm{~b}$ and $1 \mathrm{c}$. In addition, as indicated in Figure $1 \mathrm{~b}$, two spots appear strongly in the chromatograms of extracts from patients with Crohn's disease. In ulcerative colitis these two spots were either not seen or were faintly present, as illustrated in Figure 1c. The relative position of travel of these spots on the chromatogram and their staining characteristics suggested a disaccharide ceramide (probably lactosylceramide). This was confirmed by Dr B A Macher who reported that a sample of this lipid eluted from a chromatogram of resected mucosa from a patient with Crohn's disease, "clearly comigrated' with lactosylceramide which had been isolated from human neutrophils. A sample of purified lactosylceramide, supplied by Dr Macher, gave identical double spots on chromatography and co-migrated, in a range of solvent systems, with the two spots observed in Crohn's disease.

Figure 1d illustrates a chromatogram of the lipid extract from approximately $18 \times 10^{6}$ neutrophils isolated from normal human peripheral blood. Lactosylceramide can be seen to be 'strongly present' in the same relative position as that seen in Crohn's disease (Fig. 1b).

Figure 2 shows the accumulated data from our initial study of 32 patients. Lactosylceramide was not detected in extracts from the control patients nor from two cases of ulcerative colitis. The majority of samples from patients with ulcerative colitis showed a 'faintly present' lactosylceramide double spot whereas in nine of the 10 cases of Crohn's disease the double spot was 'strongly present'. All samples from the terminal ileum in Crohn's disease showed strongly present spots; the faintly present sample came from the left colon.

Lactosylceramide was not detected by chromatography of lipid extracts from uninvolved areas of the bowel in patients with Crohn's disease nor from patients with coeliac disease nor from those cases with microscopic colitis.



Fig. 2 Association of the presence and appearance of lactosylceramide with disease state.

The LC/PE ratio provided by scanning densitometry, was plotted for each of the 33 patients after breaking the code to determine the appropriate diagnostic category (Fig. 3). The LC/PE ratios (median 0.57 , range $0.3-1.57$ ) in patients with

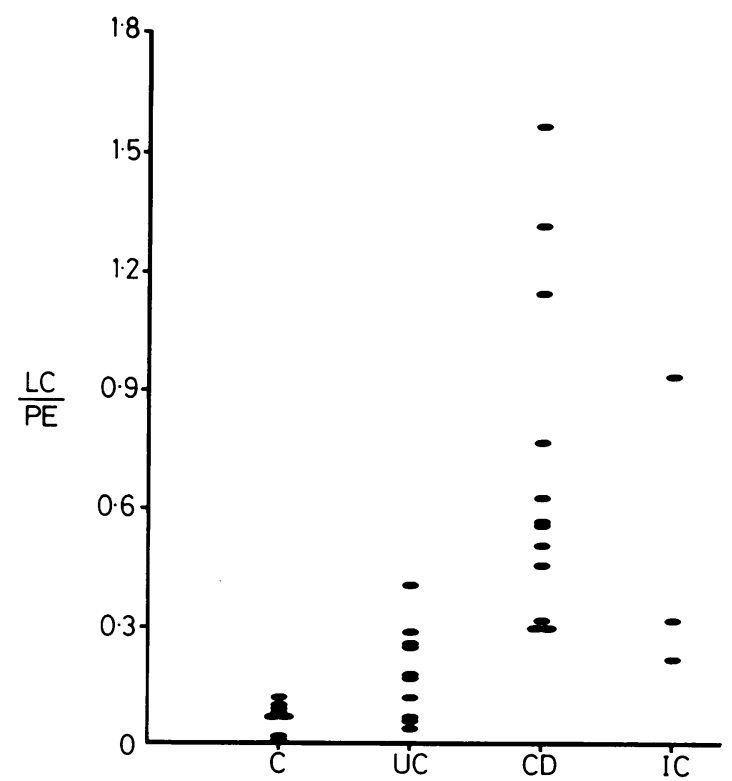

Fig. 3 Comparison of the LC/PE ratio in inflammatory bowel disease groups and in controls. $C=$ controls, $U C=$ ulcerative colitis, $C D=$ Crohn's disease, $I C=$ indeterminate colitis. 
Crohn's disease were significantly higher than the ratios (median $0 \cdot 18$, range $0 \cdot 04-0 \cdot 41$ ) in patients with ulcerative colitis $(\mathrm{p}<0.01)$. The ratios in the control patients (median $0 \cdot 08$, range $0 \cdot 01-0 \cdot 12$ ) were significantly lower than those of Crohn's disease $(p<0.01)$ and ulcerative colitis $(p=0.05)$. The three lowest results in the Crohn's disease group were from colonic biopsies. The single rectal sample gave a $\mathrm{LC} / \mathrm{PE}$ ratio of $1 \cdot 15$.

The values for the three patients with an indeterminate colitis $(0 \cdot 94,0 \cdot 32$, and $0 \cdot 22)$ are also indicated in Figure 3.

Medication and ESR at time of biopsy or surgery bore no obvious relationship to the pattern of the results, neither did disease duration.

Lactosylceramide/phosphatidylethanolamine ratio values were reproducible to between $98 \%$ and $102 \%$ of the mean value of five dilutions of the same extract. Phosphatidylethanolamine and lactosylceramide densitometer values increased in direct proportion to increasing extract concentration and to increasing volume of extract applied. Separate processing of equal aliquots of the same homogeneous sample of neutrophils resulted in lactosylceramide and phosphatidylethanolamine values which fell in a range between $94 \%$ and $106 \%$ of the mean value.

\section{Discussion}

Lipid analysis of mucosal extracts from patients with inflammatory bowel disease unexpectedly showed increased concentrations of twin spots on the lipid chromatogram in Crohn's disease. The double spot appeared to be identical to that produced by the glycosphingolipid lactosylceramide and this was confirmed by comparison with the purified compound supplied by Dr Macher. A quantitative measurement of the lactosylceramide when expressed as a ratio to phosphatidylethanolamine gave significantly higher values for patients diagnosed as Crohn's disease when compared with a group diagnosed as ulcerative colitis and to a control group.

In this study active disease sites were chosen for analysis. No patients with ulcerative colitis had active disease in the ileal region, in contrast with patients with Crohn's disease, and so such areas were not studied. It is unlikely that uninvolved terminal ileum in ulcerative colitis would show increased lactosylceramide concentrations although it would be of interest to study cases of definite ulcerative colitis which show inflammation in the ileal region. There is a suggestion that lactosylceramide concentrations in Crohn's disease may be lower in the colon than in the terminal ileum and rectum, although numbers are small.

The technique used provided a reproducible system for lipid analysis on small amounts of tissue and the use of an internal ratio to express lactosylceramide levels minimised processing variables between samples since in any one extract, as phosphatidylethanolamine and lactosylceramide should be subject to the same errors. Good reproducibility was shown using lipid extracts from blood neutrophil samples but it was not possible to investigate the variation of lactosylceramide measurements from the inflamed mucosa of individuals with inflammatory bowel disease because of the limits of tissue availability. We would predict some degree of variability between samples from patients with Crohn's disease, however, because of the associated patchy nature of inflammation. ${ }^{34}$ This would tend to produce lower, rather than higher, concentrations of lactosylceramide and therefore reduce the difference between ulcerative colitis and Crohn's disease.

Glycosphingolipids are a chemically well defined class of membrane molecules, the majority of which occupy and confer rigidity to the outer bilayer of plasma membranes, regulating membrane fluidity, ${ }^{14}$ membrane receptor protein function, ${ }^{15}$ and cell adhesion. ${ }^{1617}$ Lactosylceramide is involved in the metabolism of the more complex glycosphingolipids and is present in low concentrations in normal areas of colon from adults with adenocarcinoma, ${ }^{18}$ and normal rat small intestine. ${ }^{17}$ It is a simple glycosphingolipid having glucose and galactose in glycosidic linkage with a long chain aliphatic amine base coupled to fatty acid chains of variable length and appears as two spots on the chromatogram because the fatty acid chains exist mainly as two lengths, $\mathrm{C}_{16}$ to $\mathrm{C}_{18}$ and as $\mathrm{C}_{22}$ to $\mathrm{C}_{24} \cdot{ }^{12}$ The lactosylceramide complement of a specific tissue is made up of a characteristic mixture of slightly differing 'lactosylceramides' ${ }^{\prime 3}$ athough such differences may not be apparent on chromatography. In this study lactosylceramide could not be detected in the mucosa of control patients by visual means but low measurements were obtained using scanning densitometry. It would appear that in Crohn's disease either a novel source of lactosylceramide is present in the mucosa or normal glycosphingolipid metabolism has been altered.

Lactosylceramide has been reported to be increased in a range of other disorders including Niemann-Pick disease type $C,{ }^{19}$ familial hypercholesterolemia ${ }^{20}$ human colonic adenocarcinoma ${ }^{1 \mathrm{x}}$ and other tumours, ${ }^{21}$ and virally transformed cell lines. 22.3

In Niemann-Pick disease type $\mathrm{C}$ there is a marked decrease in lysosomal lactosylceramide galactosyl hydrolase activity resulting in lactosylceramide accumulation in hepatocytes and macrophages. Further biochemical analysis is required to investi- 
gate the possibility of a similar, but local, aberration in Crohn's disease. Serum cholesterol concentrations are lower in Crohn's disease than in normal subjects ${ }^{24}$ indicating no direct relationship between serum cholesterol and lactosylceramide concentrations. There was no evidence of adenocarcinoma in any of the patients studied and the observation that there is an increased risk of developing cancer in Crohn's disease may not be relevant as a similar risk is described in ulcerative colitis ${ }^{25}$ and the increased concentration of lactosylceramide is described after, not before, carcinoma development. It has often been postulated that Crohn's disease has an infective aetiology but to connect the increased lactosylceramide concentration with a possible viral cause would be premature in the absence of additional evidence.

A difference between the previously described examples of raised lactosylceramide concentrations and Crohn's disease is that none of the former involve an inflammatory process. Neutrophils contain high concentrations of lactosylceramide and thus acutely inflamed tissue should show higher levels of lactosylceramide than non-inflamed tissue. Inflamed areas in Crohn's disease were indeed found to contain higher amounts of lactosylceramide than non-inflamed regions, and this might be explained by the concentration of infiltrating neutrophils. There should therefore be significantly more neutrophils in Crohn's disease than ulcerative colitis, however, if they are the source of the increase in lactosylceramide. Current evidence suggests that neutrophils are involved to a similar extent in both diseases, ${ }^{20-29}$ but objective quantitative data of mucosal neutrophil density is required to confirm this.

The reason for the increase in lactosylceramide is thus unclear, but it does offer some discrimination between ulcerative colitis and Crohn's disease. A wide range of disease controls are required to establish if other conditions, for example, adenocarcinoma and infective colitides, show raised lactosylceramide concentrations, however, as mucosal tissue is required for the test, histological examination with its range of differential diagnoses remains the method of choice. Lactosylceramide measurement may be useful in the diagnosis of indeterminate colitis, where histology cannot make a clear distinction between Crohn's disease and ulcerative colitis. The lactosylceramide to phosphatidylethanolamine ratio measurement produced an overlap between the two diseases, and one case of indeterminate colitis fell within this range. The other two cases gave probable diagnoses of Crohn's disease and ulcerative colitis respectively. It remains to be seen if these predicted diagnoses are correct. Lactosylceramide measurement may be of more general use if the mucosal results are paralleled in samples of urine and/or faeces as it may then act as a simple screening test.

This work was funded by the Crohn's in Childhood Research Appeal (CICRA). We would like to thank Miss Jane Sandall and the Imperial Cancer Research Fund, Lincoln Inn's Field, for supplying scanning densitometry facilities. We also thank surgical and gastroenterological colleagues at St Bartholomew's Hospital for providing tissue samples. Part of this work was published in abstract form in Gut 1985; 26: A1156.

\section{References}

1 Lewis D, Walker-Smith JA, Phillips AD. Microvilli- and desmosome-associated bodies in Crohn's disease and other disorders in childhood: An ultrastructural abnormality of the small intestine. J Pediatr Gastroenterol Nutr 1984; 3: 46-55.

2 Chong SKF, Bartram C, Campbell CA, Williams CB, Blackshaw AJ, Walker-Smith JA. Chronic inflammatory bowel disease in childhood. $B r$ Med J 1982; 284: $101-4$.

3 Lockhart-Mummery HE, Morson BC. Crohn's disease (regional enteritis) of the large intestine and its distinction from ulcerative colitis. Gut 1960; 1: 87-105.

4 Morson BC, Dawson IMP. Crohn's disease. Gastrointestinal pathology. London: Blackwell Scientific Publications, 1979: 293-312.

5 Chong SKF, Blackshaw AJ, Boyle S, Williams CB, Walker-Smith JA. Histological diagnosis of chronic inflammatory bowel disease in childhood. Gut 1985; 26: 55-9.

6 Ferrante A, James DW, Betts WH, Cleland LG. Rapid single-step method for purification of polymorphonuclear leucocytes from blood of patients with rheumatoid arthritis. Clin Exp Immunol 1982; 47: 749-52.

7 Bligh EG, Dyer WJ. A rapid method of total lipid extraction and purification. Can J Biochem Physiol 1959; 37: $911-7$.

8 Sheltawy A, Dawson RMC. Separation and estimation of phospholipids. In: Smith I, ed. Chromatographic and electrophoretic techniques. Vol. 1. London: Heinnemann Medical Books Ltd, 1969: 450-93.

9 Owens K. A two-dimensional thin-layer chromatographic procedure for the estimation of plasmalogens. Biochem J 1965; 100: 354-61.

10 Rouser G, Fleischer S, Yamamoto A. Two dimensional thin layer chromatographic separation of polar lipids and determination of phospholipids by phosphorus analysis of spots. Lipids 1970; 5: 494-6.

11 Heymans HSA, Bosch $\mathrm{H}$, Schutgens $\mathrm{RBH}$, et al. Deficiency of plasmalogens in the cerebro-hepato-renal (Zellweger) syndrome. Eur J Pediatr 1984; 142: 10-5.

12 Macher BA, Klock JC. Isolation and characterisation of neutral glycosphingolipids of human neutrophils. $J$ Biol Chem 1985; 255: 2092-6.

13 Fukuda MN, Dell A, Oates JE, Wu P, Klock JC, Fukuda M. Structures of glycosphingolipids isolated from human granulocytes. J Biol Chem 1985; 260: 1067-82. 
14 Yamakawa T, Nagai Y. Glycolipids at the cell surface and their biological significance. Trends Biochem Sci 1978; 3: 128-31.

15 Hakomori S-I. Glycosphingolipids in cellular interaction, differentiation, and oncogenesis. Ann Rev Biochem 1981; 50: 733-64.

16 Glickman RM, Bouhours JF. Characterisation, distribution and biosynthesis of the major gangliosides of rat intestinal mucosa. Biochim Biophys Acta 1976; 424: 17-25.

17 Bouhours JF, Glickman RM. Rat intestinal glycolipids. II Distribution and biosynthesis of glycolipids and ceramide in villus and crypt cells. Biochim Biophys Acts 1976; 441: 123-33.

18 Siddiqui B, Whitehead JS, Kim YS. Glycosphingolipids in human colonic adenocarcinoma. J Biol Chem 1978; 253: 2168-75.

19 Vanier MT. Biochemical studies in Niemann-Pick disease. 1. Major sphingolipids of liver and spleen. Biochim Biophys Acta 1983; 750: 178-84.

20 Chattergee S, Clarke KS, Kwiterovich PO. Uptake and metabolism of lactosylceramide on low density lipoproteins in cultured proximal tubular cells from normal and familial hypercholesterolemic homozygotes. J Biol Chem 1986; 261: 13480-6.

21 Kanazawa I, Yamakawa T. Presence of glucosylcera- mide and lactosylceramide in human intracranial tumors. Jpn J Exp Med 1974; 44: 379-84.

22 Hakomori S-I, Murakami WT. Glycosphingolipids of hamster fibroblasts and derived malignant-transformed cell lines. Proc Natl Acad Sci USA 1968; 59: 254-61.

23 Den H, Sela BA, Roseman S, Sachs L. Blocks in ganglicside synthesis in transformed hamster cells and their revertants. J Biol Chem 1974; 249: 659-61.

24 Rutgeers P, Ghoos Y, Vantrappen G, Fevery J. Biliary lipid composition in patients with nonoperated Crohn's disease. Dig Dis Sci 1986; 31: 27-32.

25 Glotzer DJ. The risk of cancer in Crohn's disease. Gastroenterology 1985; 89: 438-41.

26 Saverymuttu SH, Peters AM, Lavender JP, Hodgson HJ, Chadwick VS. 111-Indium autologous leucocytes in inflammatory bowel disease. Gut 1983; 24: 293-9.

27 Kane SP, Hoffbrand AV, Neal EG. Indices of granulocyte activity in inflammatory bowel disease. Gut 1974; 15: 953-9.

28 Otto HF, Gebbers JO. Electron microscopic, ultracytochemical and immunohistological observations in Crohn's disease of the ileum and colon. Virchows Arch (A) 1981; 391: 189-205.

29 Lewis DC, Walker-Smith JA, Phillips AD. Polymorphonuclear neutrophil leucocytes in childhood Crohn's disease: a morphological study. J Pediatr Gastroenterol Nutr 1987; 6: 430-8. 